\title{
Metabolomics of an in vitro liver model containing primary hepatocytes assembling around an endothelial cell network: comparative study on the metabolic stability and the effect of acetaminophen treatment
}

\author{
Yu Toyoda1,2, Kasumi Kashikura ${ }^{3}$, Tomoyoshi Soga ${ }^{3}$ and Yoh-ichi Tagawa²,4 \\ 'Department of Pharmacy, The University of Tokyo Hospital, 7-3-1 Hongo, Bunkyo-ku, Tokyo 113-8655, Japan \\ ${ }^{2}$ Department of Biomolecular Engineering, Graduate School of Bioscience and Biotechnology, \\ Tokyo Institute of Technology, 4259 Nagatsuta-cho, Midori-ku, Yokohama 226-8501, Japan \\ ${ }^{3}$ Institute for Advanced Biosciences Keio University, 246-2 Mizukami, Kakuganji, Tsuruoka, Yamagata 997-0052, Japan \\ ${ }^{4}$ Department of Life Science and Technology, School of Life Science and Technology, Tokyo Institute of Technology, \\ 4259 Nagatsuta-cho, Midori-ku, Yokohama 226-8501, Japan
}

(Received March 6, 2017; Accepted April 23, 2017)

\begin{abstract}
Recently, a novel culture system consisting of primary hepatocytes structured over a network of endothelial cells on the Engelbreth-Holm-Swarm (EHS) gel has been reported. This in vitro liver model on the EHS gel ( $\mathrm{IVL}_{\mathrm{EHS}}$ ) has been shown to maintain the expression of hepatic genes and their functional activity. Moreover, the $\mathrm{IVL}_{\mathrm{EHS}}$ was more sensitive to xenobiotics than hepatocyte monocultures, suggesting the potential utility of this culture system for compound hepatotoxicity screening. However, the effect of this three-dimensional structure formation on the cellular metabolic profile of hepatocytes in the $\mathrm{IVL}_{\mathrm{EHS}}$ is not well understood. To address this concern, we performed metabolome analysis using capillary electrophoresis-time of flight mass spectrometry. Between the $\mathrm{IVL}_{\mathrm{EHS}}$ and mono-cultured hepatocytes on the EHS gel, there was no significant difference in the levels of metabolites of the urea cycle and the tricarboxylic acid cycle, essential amino acids, and adenylate energy charge (AEC) which is an important indicator of cellular energy status. On the other hand, acetaminophen-dependent decrease of the $\mathrm{AEC}$ in the $\mathrm{IVL}_{\mathrm{EHS}}$ was greater than that in the monoculture, suggesting the higher sensitivity of $\mathrm{IVL}_{\mathrm{EHS}}$ to acetaminophen-induced hepatotoxicity which is caused by metabolic activation of this drug. Further analysis showed that the levels of taurocholate, one of the major conjugated bile acids, were higher in the $\mathrm{IVL}_{\mathrm{EHS}}$ than in the monoculture. Considering that the construction of the $\mathrm{IVL}_{\mathrm{EHS}}$ did not seem to disturb the major cellular metabolism, our findings would strengthen the concept that $\mathrm{IVL}_{\mathrm{EHS}}$ would have beneficial effects on the maintenance of hepatic functions.
\end{abstract}

Key words: Acetaminophen, Adenylate energy charge, CE-TOFMS, EHS gel, Hepatotoxicity, In vitro liver model (IVL)

\section{INTRODUCTION}

The liver is the largest internal organ performing such multiple vital functions as metabolic conversion of xenobiotics, protein synthesis, carbohydrate storage, and bile secretion. Hitherto, in vitro liver models faithfully reproducing hepatic functions have been extensively investigated with the aim of developing alternatives to liver transplantation (Sharma et al., 2010; Viollet et al., 2009). Moreover, such in vitro liver-mimicking systems are expected to be used for compound screening to predict potential hepatotoxicity as well as pharmacological effects of drug discovery (Hewitt et al., 2007; Kaplowitz, 2005). One of the major approaches in liver modeling has been the use of primary hepatocytes, which constitute about two thirds of the total cell population in the liver parenchyma (Godoy et al., 2013; Donato et al., 2013; Guguen-Guillouzo and Guillouzo, 2010). However, the use of primary hepatocytes is challenging, because the hepatocytes rapidly lose their function after isolation, and

Correspondence: Yoh-ichi Tagawa (E-mail: ytagawa@bio.titech.ac.jp) 
their maintenance in simple monoculture condition is difficult.

Considering that the liver is composed of parenchymal hepatocytes as well as of non-parenchymal cells, the structural arrangement of hepatic tissue should be important for supporting the functional activity of hepatocytes (Godoy et al., 2013). It has been established that the survival and functional performance of primary hepatocytes in vitro critically depend on the intercellular communication of hepatocytes with the adjacent non-parenchymal cells as well as on the interaction between hepatocytes (Kidambi et al., 2009; Nahmias et al., 2006; Strain, 1999; Bhatia et al., 1999, 1998). In addition to cell-cell contacts, the presence of the extracellular matrix (ECM) is also important, as the reconstitution of the ECM in vitro contributes to the maintenance of cell morphology and functional integrity (Gissen and Arias, 2015; Kleinman et al., 2003; DiPersio et al., 1991). When hepatocytes are cultured on ECM-derived gels containing type I collagen or basement membrane proteins, they retain their differentiated morphology for longer time and express liver-specific genes at a higher level compared to monolayer cultures on plastic surface, which have flattened shape and rapidly lose their liver-specific characteristics (Schuetz et al., 1988). In this context, spatially coordinated interaction of non-parenchymal cells and the ECM with hepatocytes would be critical for the in vitro maintenance of primary hepatocyte cultures and induction of their liverspecific functions.

We have recently developed an in vitro liver model (Toyoda et al., 2012) consisting of primary hepatocytes and endothelial cells cultured on the Engelbreth-HolmSwarm (EHS) gel, an extract of basement membrane proteins (Kleinman and Martin, 2005). In this model called the $\mathrm{IVL}_{\mathrm{EHS}}$, primary hepatocytes gather and assemble in a three-dimensional manner around a capillary-like tubular network of endothelial cells plated on the EHS gel, resulting in a structure that resembles hepatic tissue. In the presence of acetaminophen, an inducer of metabolic conversion-related hepatotoxicity (Coen, 2015; Jaeschke et al., 2003), the $\mathrm{IVL}_{\mathrm{EHS}}$ tissue model was reportedly more sensitive to acetaminophen-induced toxicity compared to monolayer cultures (Toyoda et al., 2012). Furthermore, the previous study showed that the $\mathrm{IVL}_{\mathrm{EHS}}$ maintained the drug-metabolizing capacity of primary hepatocytes as evidenced by the expression of liver-specific drug-metabolizing enzymes and biliary efflux transporters, and the higher cytochrome P450 activities as compared with those in the mono-culture conditions.

Whereas the potential utility of the $\mathrm{IVL}_{\mathrm{EHS}}$ has been pointed out (Ahn et al., 2014; Toyoda et al., 2012), there is little knowledge about the endogenous metabolism of hepatocytes in the IVL $\mathrm{EHS}_{\mathrm{EHS}}$ such as ammonia detoxification in the urea cycle and general pathways for energy generation. Since environmental changes can disrupt intracellular homeostasis of cells and cause subsequent alterations in gene expression (de Nadal et al., 2011; Viollet et al., 2009; Dalton et al., 1999), it would be important to clarify whether the metabolic profile of hepatocytes is affected by the construction of IVL $_{\mathrm{EHS}}$ characterized by spontaneous formation of three-dimensional structure and hepatocyte migration on the EHS gel.

In this study, we constructed the $\mathrm{IVL}_{\mathrm{EHS}}$ according to the previous study (Toyoda et al., 2012) to enhance our understanding about the biochemical characteristics of this culture system. The effects of the construction of $\mathrm{IVL}_{\mathrm{EHS}}$ were examined on the cellular metabolism of hepatocytes using metabolomics approach. There was no significant difference in the levels of metabolites of the urea cycle and the tricarboxylic acid (TCA) cycle as well as in adenylate energy charge (AEC) between the $\mathrm{IVL}_{\mathrm{EHS}}$ and monocultures of hepatocytes on the EHS gel, suggesting that the IVL $\mathrm{EHS}_{\mathrm{EHS}}$ could preserve hepatic functions without negative effects on hepatocyte metabolism.

\section{MATERIALS AND METHODS}

\section{Materials}

The following reagents were used: endothelial cell basal medium (EBM-2) and EGM-2 Single Quots (supplemental factors) (Lonza Japan Ltd., Tokyo, Japan); Dulbecco's modified Eagle's medium (DMEM), penicillin/streptomycin $(100 \times)$, and trypsin-EDTA (Invitrogen/GIBCO, Grand Island, NY, USA); tissue-culture treated cell culture dishes/plates (Corning Inc., Tokyo, Japan); collagenase, trypsin inhibitor, mannitol, D-camphor-10-sulfonic acid (CSA), 1,3,5-benzenetricarboxylic acid (Trimesate), and gelatin (Wako Pure Chemical Industries Ltd., Osaka, Japan); 2-(N-morpholino) ethanesulfonic acid (MES) (Dojindo, Kumamoto, Japan); growth factor reduced (GFR) Engelbreth-Holm-Swarm (EHS) gel (BD Biosciences, Bedford, MA, USA); and acetaminophen (p-Acetamidophenol) (Nacalai Tesque Inc., Kyoto, Japan). All reagents used in the present study were of analytical grade.

\section{Animals}

Six-week-old male BALB/cAJc1 mice were purchased from CLEA Japan Inc. (Tokyo, Japan) and treated in accordance with local institutional guidelines for the care and use of laboratory animals. The animal protocol was approved by the Animal Experimentation Committee of 


\section{Metabolomics of an in vitro liver model}

Tokyo Institute of Technology.

\section{Primary cell culture}

Human umbilical vein endothelial cells (HUVECs) (Cambrex BioScience Walkersville Inc., Walkersville, MD, USA) were maintained at $37^{\circ} \mathrm{C}$ in a humidified atmosphere of $5 \% \mathrm{CO}_{2}$ in air in complete EBM-2 containing $2 \%(\mathrm{v} / \mathrm{v})$ fetal bovine serum, hydrocortisone, hFGF- $\beta$, VEGF, R3-IGF-1, ascorbic acid, hEGF, GA-1000, and heparin according to the manufacturer's instructions. Cells were subcultured not more than 10 times. Mouse primary hepatocytes were isolated by the in situ 2-stepcollagenase perfusion method via portal vein cannulation as described in previously (Toyoda et al., 2012). In brief, after pre-perfusion with Hank's Buffered Salt Solution (HBSS) containing EGTA $(0.19 \mathrm{~g} / \mathrm{L})$ and glucose $(0.98 \mathrm{~g} / \mathrm{L})$, the mouse liver was perfused with $0.015 \%$ collagenase in HBSS, then minced in ice-cold HBSS. To isolate the hepatocytes, the dispersed cells were filtered, and subjected to $40 \%$ Percoll density gradient centrifugation. Isolated hepatocytes were maintained in DMEM supplemented with $100 \mathrm{U} / \mathrm{mL}$ penicillin and $100 \mu \mathrm{g} / \mathrm{mL}$ streptomycin at $37^{\circ} \mathrm{C}$ in a humidified atmosphere of $5 \%$ $\mathrm{CO}_{2}$ in air.

\section{Construction of the $\mathrm{IVL}_{\mathrm{EHS}}$}

The IVL $_{\mathrm{EHS}}$, an in vitro liver model containing primary hepatocytes assembled around an endothelial cell network on the EHS gel, was constructed according to our previous study (Toyoda et al., 2012). HUVECs $\left(3.5 \times 10^{5}\right.$ cells/well of 6-well plates) were seeded on the EHS gel, and the endothelial cell network was obtained after $3 \mathrm{hr}$. Four hours after HUVEC seeding, freshly isolated mouse primary hepatocytes $\left(1.0 \times 10^{6}\right.$ cells/well of 6 -well plates $)$ were seeded on the HUVEC network structure in $10 \%$ FBS-containing medium and allowed to attach; after $4 \mathrm{hr}$, the medium was replaced with fresh medium. The experiments were started $24 \mathrm{hr}$ after seeding (time 0 ), when almost all primary hepatocytes completed the migration toward the network and piled up. At that time, medium was changed to fresh one with or without $10 \mathrm{mM}$ acetaminophen, since the previous study showed that this dose of acetaminophen was enough to induce hepatotoxicity in the IVL $_{\mathrm{EHS}}$ (Toyoda et al., 2012). Additionally, these common experimental conditions allowed us to interpret the obtained data based on the previous study. Culture medium collected at each time point was centrifuged for $5 \mathrm{~min}$ at $300 \times g$ to remove floating cells, and supernatant was stored at $-80^{\circ} \mathrm{C}$ until use.

\section{Albumin detection by ELISA}

Mouse albumin in culture medium was detected using the Mouse Microalbuminuria ELISA kit (Albuwell M; Exocell, Philadelphia, PA, USA) according to the manufacturer's introduction. Briefly, $50 \mu \mathrm{L}$ of culture supernatant and equal volume of rabbit anti-mouse albumin antibody solution were added to the albumin-coated test plate for $30 \mathrm{~min}$ at room temperature. After washing 10 times with rinse solution, an anti-rabbit HRP-conjugated antibody $(100 \mu \mathrm{L}$ per well) was added for $30 \mathrm{~min}$. After washing 10 times, $100 \mu \mathrm{L}$ of Color Development solution per well was added for $5 \mathrm{~min}$; the chromogenic reaction was stopped by an equal volume of Color Stopper solution and evaluated at $450 \mathrm{~nm}$ using the iMark $^{\text {TM }}$ Microplate Reader. Albumin concentration in each sample was calculated according to a standard curve.

\section{Urea assay}

Urea concentration in culture medium was determined by using the QuantiChrom ${ }^{\mathrm{TM}}$ Urea Assay Kit (BioAssay Systems, Hayward, CA, USA) according to the manufacturer's instruction. In brief, $5 \mu \mathrm{L}$ of culture medium was incubated with $200 \mu \mathrm{L}$ of the reaction mixture for $30 \mathrm{~min}$ at room temperature, and urea-dependent chromogenic reaction was evaluated at $492 \mathrm{~nm}$ using an iMark $^{\mathrm{TM}}$ Microplate Reader (Bio-Rad Laboratories, Inc., Hercules, CA, USA). Urea concentration in each sample was calculated based on a standard curve constructed using known concentrations of urea.

\section{CE-TOFMS analysis}

Intracellular metabolites were analyzed $12 \mathrm{hr}$ after $\mathrm{IVL}_{\mathrm{EHS}}$ construction the capillary electrophoresis (CE)time of flight-mass spectrometry (TOFMS) method as described previously (Soga et al., 2006). Briefly, cells on the EHS gel were washed twice, treated with 5\% mannitol, and saturated with $1 \mathrm{~mL}$ of $\mathrm{MeOH}$ containing $25 \mu \mathrm{M}$ of each internal standard (MES and CSA) for $10 \mathrm{~min}$; the extraction was performed at $4^{\circ} \mathrm{C}$ to minimize sample degradation and halt potential enzymatic reactions during sample preparation. The resulting solution was immediately mixed with $400 \mu \mathrm{L} \mathrm{CHCl}_{3}$ and $200 \mu \mathrm{L}$ water, and centrifuged at $10,000 \times \mathrm{g}$ for $3 \mathrm{~min}$ at $4^{\circ} \mathrm{C}$. Subsequently, the $400-\mu \mathrm{L}$ upper aqueous layer was filtered through a Millipore 5-kDa cutoff filter by centrifugation to remove proteins. The filtrate was lyophilized and dissolved in $50 \mu \mathrm{L}$ water containing reference compounds (3-aminopyrrolidine and Trimesate) prior to CETOFMS analysis. The extracts were obtained from the $\mathrm{IVL}_{\mathrm{EHS}}$ and hepatocyte monocultures grown on the EHS gel (control), which were treated or not with acetami- 
nophen $(10 \mathrm{mM})$ for $12 \mathrm{hr}$.

CE-TOFMS was performed using an Agilent CE capillary electrophoresis system, Agilent G3250AA LC/ MSD TOF system, Agilent 1100 series binary HPLC pump, G1603A Agilent CE-MS adapter, and G1607A Agilent CE-ESI-MS sprayer kit (Agilent Technologies, Waldbronn, Germany) according to previous studies (Soga et al., 2002, 2003, 2006, 2009; Hirayama et al., 2009). For system control and data acquisition, we used the G2201AA Agilent ChemStation software for CE, and the Analyst QS software for TOFMS.

\section{Data normalization}

The levels of metabolites in each sample were normalized to that of mouse albumin in culture medium at the time of metabolite extraction for CE-TOFMS analysis according to previous study (Tsutsui et al., 2006), since the amount of cellular protein could not be determined because of the presence of the EHS gel. Our previous study demonstrated that albumin expression levels in primary hepatocytes were hardly affected by the presence of EHS gel and/or HUVECs $48 \mathrm{hr}$ after the seeding (Toyoda et al., 2012). Thus, we focused on the mouse albumin level in the culture medium as a detectable indicator of the number of hepatocytes in each culture system. Furthermore, our previous study showed that LDH levels in HUVECs constituted $<5 \%$ of total LDH in the $\mathrm{IVL}_{\mathrm{EH}}$, suggesting that HUVECs did not significantly contribute to the total extracts of the $\mathrm{IVL}_{\mathrm{EHS}}$ (Toyoda et al., 2012). Hence, we could reliably compare the relative levels of metabolites in $\mathrm{IVL}_{\mathrm{EHS}}$ hepatocytes with those in hepatocyte monocultures.

\section{Statistical analysis}

All statistical analyses were performed by using EXCEL (Microsoft Corp., Redmond, WA, USA) with the Statcel3 add-in software (OMS Publishing Inc., Saitama, Japan). Differences between samples were analyzed by Student's $t$ test, and considered statistically significant at the $P$ value less than 0.05 and 0.01 .

\section{RESULTS AND DISCUSSION}

\section{Albumin levels in $\mathrm{IVL}_{\mathrm{EHS}}$ culture medium}

To construct the $\mathrm{IVL}_{\mathrm{EHS}}$ model, we seeded mouse primary hepatocytes on the EHS gel with or without HUVEC network. After $\mathrm{IVL}_{\mathrm{EHS}}$ construction, the cells were cultured for further $12 \mathrm{hr}$ in the absence or presence of acetaminophen to induce hepatic injury, then the cellular extracts were collected. At the sampling time point for metabolome analysis, mouse albumin concentration in culture medium was measured in each acetaminophen condition. In the absence of acetaminophen, there was no difference in medium albumin levels between hepatocytes cultured with or without HUVECs $(1.56 \pm 0.17$ versus $1.55 \pm 0.13 \mu \mathrm{g} / \mathrm{mL}$, respectively), which is consistent with comparable albumin expression in hepatocytes cultured on the EHS gel with or without HUVECs for $48 \mathrm{hr}$ (Toyoda et al., 2012). On the other hand, in the presence of acetaminophen, albumin levels in culture medium of the $\operatorname{IVL}_{\mathrm{EHS}}(0.53 \pm 0.07 \mu \mathrm{g} / \mathrm{mL})$ were significantly lower than those of mono-cultured hepatocytes on EHS gel $(0.66 \pm 0.10 \mu \mathrm{g} / \mathrm{mL})$, suggesting higher sensitivity of hepatocytes in the $\mathrm{IVL}_{\mathrm{EHS}}$ to acetaminophen-induced injuries. Considering that acetaminophen overdose has the potential for causing hepatotoxicity and acute liver failure in vivo (Lancaster et al., 2015), together with the previously determined higher cytochrome $\mathrm{P} 450$ activities in the $\mathrm{IVL}_{\mathrm{EHS}}$ (Toyoda et al., 2012), the $\mathrm{IVL}_{\mathrm{EHS}}$ should be superior to the monoculture on EHS gel in keeping hepatocytes in a state close to the those in vivo.

\section{Metabolites in the urea cycle}

In a previous study, the urea levels in culture medium of $\mathrm{IVL}_{\mathrm{EHS}}$, total amount of cell-secreted urea, was only examined (Toyoda et al., 2012); therefore, in this study, we first focused on the major intracellular metabolites of the urea cycle. The levels of intracellular urea, ornithine, citrulline, aspartate, fumarate, and arginine were determined, whereas the levels of carbamoyl phosphate and arginosuccinate were below the detection limit (Fig. 1a). The urea cycle is regulated by carbamoyl phosphate synthetase 1 (CPS-1), a rate-limiting enzyme allosterically activated by $N$-acetylglutamate (NAG). Therefore, we examined NAG levels in each culture condition. In the absence of acetaminophen, no significant differences were observed in urea cycle metabolites between the IVL $\mathrm{EHS}_{\mathrm{EHS}}$ and monoculture on the EHS gel (control) (Fig. 1a), which was in agreement with similar concentrations of urea in the medium of the $\mathrm{IVL}_{\mathrm{EHS}}$ and control cultures (Fig. 1b). In addition, the detected urea concentrations in the culture medium of $\mathrm{IVL}_{\mathrm{EHS}}$ were comparable with those that were detected in our previous study (Toyoda et al., 2012), suggesting that $\mathrm{IVL}_{\mathrm{EHS}}$ was wellconstructed in the present study. Furthermore, NAG levels were almost the same between the $\mathrm{IVL}_{\mathrm{EHS}}$ and control. These data suggest that the construction of the $\mathrm{IVL}_{\mathrm{EHS}}$ did not affect urea metabolism in hepatocytes. Similarly, no significant differences in the urea cycle metabolites were observed between the $\mathrm{IVL}_{\mathrm{EHS}}$ and control after acetaminophen treatment, with the exception of arginine (Fig. 1a). Regarding this point, we speculate that one 
Metabolomics of an in vitro liver model

a

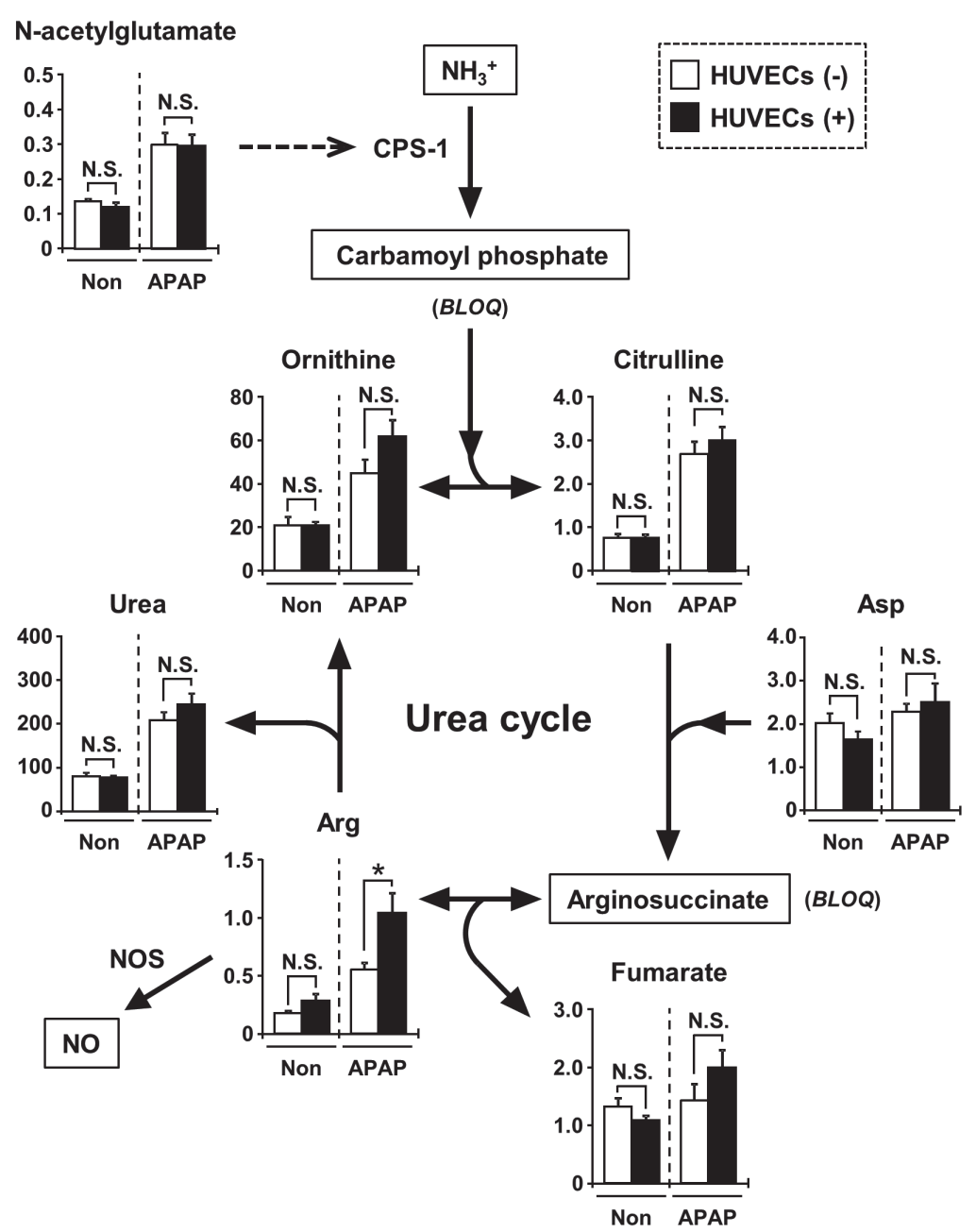

b

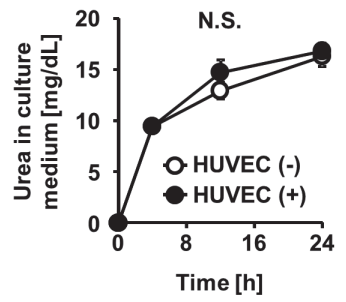

Fig. 1. Urea cycle metabolites in the $\mathrm{IVL}_{\mathrm{FHS}}$ model and monocultures. a. Levels of major urea cycle metabolites in the $\mathrm{IVL}_{\mathrm{FHS}}$ and control cultures with or without acetaminophen treatment. The y-axis shows relative metabolite levels (nmol/ $\mu \mathrm{g}$ of albu$\mathrm{min}$ ). White and black columns indicate control and $\mathrm{IVL}_{\mathrm{FHS}}$, respectively. Non, no treatment; APAP, acetaminophen; $B L O Q$, metabolite level below the limitation of quantification. Data are expressed as the mean \pm S.E.M. $n=4$. b. Time-dependent increase of urea levels in hepatocyte culture medium of the $\mathrm{IVL}_{\mathrm{EHS}}$ (with HUVEC) and monocultures (without HUVEC). Data are expressed as the mean \pm S.D. $n=3$. Statistical analyses for significant differences were performed according to Student's $t$ test $(*, P<0.05 ; * *, P<0.01$ compared to control). N.S., not significantly different among groups.

plausible explanation of the increase of arginine may be the suppression cytotoxic nitric oxide (NO) production, although we could not determine the NO levels and NO synthase activities in the $\mathrm{IVL}_{\mathrm{EHS}}$ due to the technical limitations in the present study. However, considering that there was no significant difference in the NAcGlc levels between the IVL $\mathrm{EHS}_{\mathrm{EHS}}$ and control, it is unlikely that the construction of $\mathrm{IVL}_{\mathrm{EHS}}$ could affect the overall activity of the urea cycle in $\mathrm{IVL}_{\mathrm{EHS}}$ hepatocytes. In this context, the ability for ammonia detoxification in the $\mathrm{IVL}_{\mathrm{EHS}}$ should be the almost same as in hepatocyte monocultures, supporting that these two culture systems could have similar tolerance to exogenous hepatotoxic stimuli.

\section{Adenylate energy charge and lactate/pyruvate ratio}

We next compared the energy status in the two culture systems, $\mathrm{IVL}_{\mathrm{EHS}}$ and monoculture on the EHS gel. First, we addressed the AEC by CE-TOFMS (Fig. 2a). The AEC is an important indicator of the energy state in the cells 
a

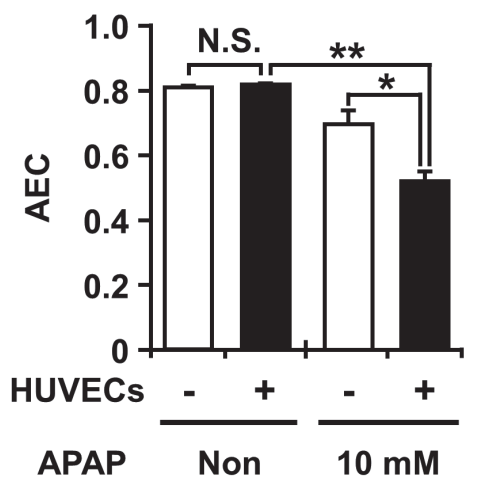

b

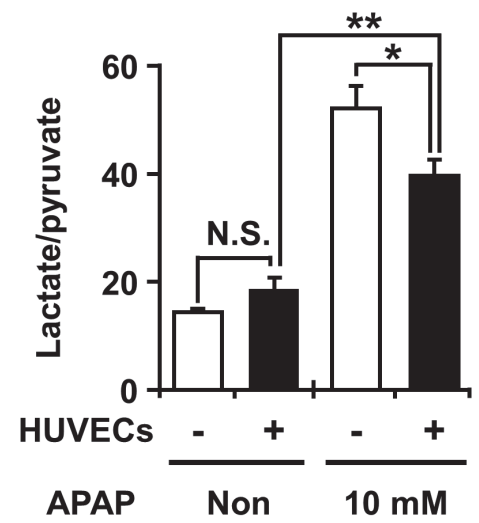

Fig. 2. Energy and redox states in hepatocytes from the $\mathrm{IVL}_{\mathrm{EHS}}$ and monocultures. Hepatocytes cultures were treated or not with $10 \mathrm{mM}$ acetaminophen (APAP) for $12 \mathrm{hr}$. a. Adenylate energy charge (AEC). b. Lactate/pyruvate (L/P) ratio. White and black columns indicate control and $\mathrm{IVL}_{\mathrm{EHS}}$, respectively. Data are expressed as the mean \pm S.E.M. $n=4$. Statistical analyses for significant differences were performed according to Student's $t$ test ( ${ }^{*}, P<0.05 ; * *, P<0.01$ compared to control). N.S., not significantly different among groups.

(Atkinson, 1968; Hirayama et al., 2009), defined by the following equation; $([\mathrm{ATP}]+0.5[\mathrm{ADP}]) /([\mathrm{ATP}]+[\mathrm{ADP}]$ $+[\mathrm{AMP}])$; accordingly, the AEC values around 1 indicate cell health, while those close to zero suggest cell damage and/or death (Zhang and Vertes, 2015). The results showed that in the absence of acetaminophen, AEC values in the two culture systems were similar $(0.815 \pm$ 0.008 in monocultures and $0.822 \pm 0.004$ in the $\mathrm{IVL}_{\mathrm{EHS}}$, respectively) (Fig. 2a). Considering that the AEC value in the liver of rodents is about 0.8 (Chapman et al., 1971), hepatocytes in the two groups were close to the normal physiological state. However, acetaminophen treatment decreased AEC values to $0.699 \pm 0.089$ in control (monocultures) and to $0.522 \pm 0.062$ in the $\operatorname{IVL}_{\mathrm{EHS}}$ (Fig. 2a), indicating the presence of hepatic injury. The decrease of AEC was more significant in the IVL $\mathrm{E}_{\mathrm{EHS}}$, which should reflect higher sensitivity of the $\mathrm{IVL}_{\mathrm{EHS}}$ to acetaminophen compared with hepatocyte monocultures.

We then evaluated the ratio of free NAD to NADH (NAD/NADH) indicative of cellular redox status (Williamson et al., 1967), by measuring the lactate/pyruvate ( $\mathrm{L} / \mathrm{P}$ ratio). In the absence of acetaminophen, there was no significant difference in the $\mathrm{L} / \mathrm{P}$ ratio between the $\mathrm{IVL}_{\mathrm{EHS}}$ and control cultures (Fig. 2b), suggesting that $\mathrm{IVL}_{\mathrm{EHS}}$ construction did not negatively affect the redox status in hepatocytes. The balance between free NAD and $\mathrm{NADH}$ in the cytosol plays a pivotal role in the regulation of cellular redox homeostasis and, thus, could be used to assess cellular metabolic state (Sun et al., 2012; Debray et al., 2007; Lin and Guarente, 2003). However, there are technical difficulties in distinguishing between proteinbound and free NAD/NADH; therefore, it has been generally accepted that free NAD/NADH should be estimated based on the L/P ratio according to the principle of chemical equilibrium (pyruvate $+\mathrm{NADH} \leftrightarrow$ lactate + NAD) (Debray et al., 2007; Williamson et al., 1967). For example, when cellular respiration is impaired, pyruvate oxidation is decreased and the reduced form of redox coenzyme NADH would be predominant (Debray et al., 2007), i.e., the ratio of $\mathrm{L} / \mathrm{P}$ would increase and that of NAD/NADH decrease. Consistent with this context, acetaminophen treatment increased the $\mathrm{L} / \mathrm{P}$ ratio both in the $\mathrm{IVL}_{\mathrm{EHS}}$ and in control cultures (Fig. 2b); however, the increasing effect was lowers in the IVL $\mathrm{EHS}_{\mathrm{EHS}}$ than in control. While the $\mathrm{IVL}_{\mathrm{EHS}}$ was more sensitive to acetaminophen than monoculture control in terms of energy status (Fig. 2a), these results imply that the construction of the $\mathrm{IVL}_{\mathrm{EHS}}$ could enhance molecular mechanism(s) involved in the maintenance of cellular redox potential in response to exogenous damage.

\section{Metabolites of the glycolysis and TCA pathways}

We focused on glycolysis and the TCA cycle, which are major pathways regulating cellular energy metabolism. The pathways and relative levels of their major metabolites are presented in Fig. 3. In the absence of acetaminophen, there was no significant difference in the levels of most metabolites between the $\mathrm{IVL}_{\mathrm{EHS}}$ and control, suggesting comparable ability for energy generation, which is consistent with similar AEC levels (Fig. 2a). Among 


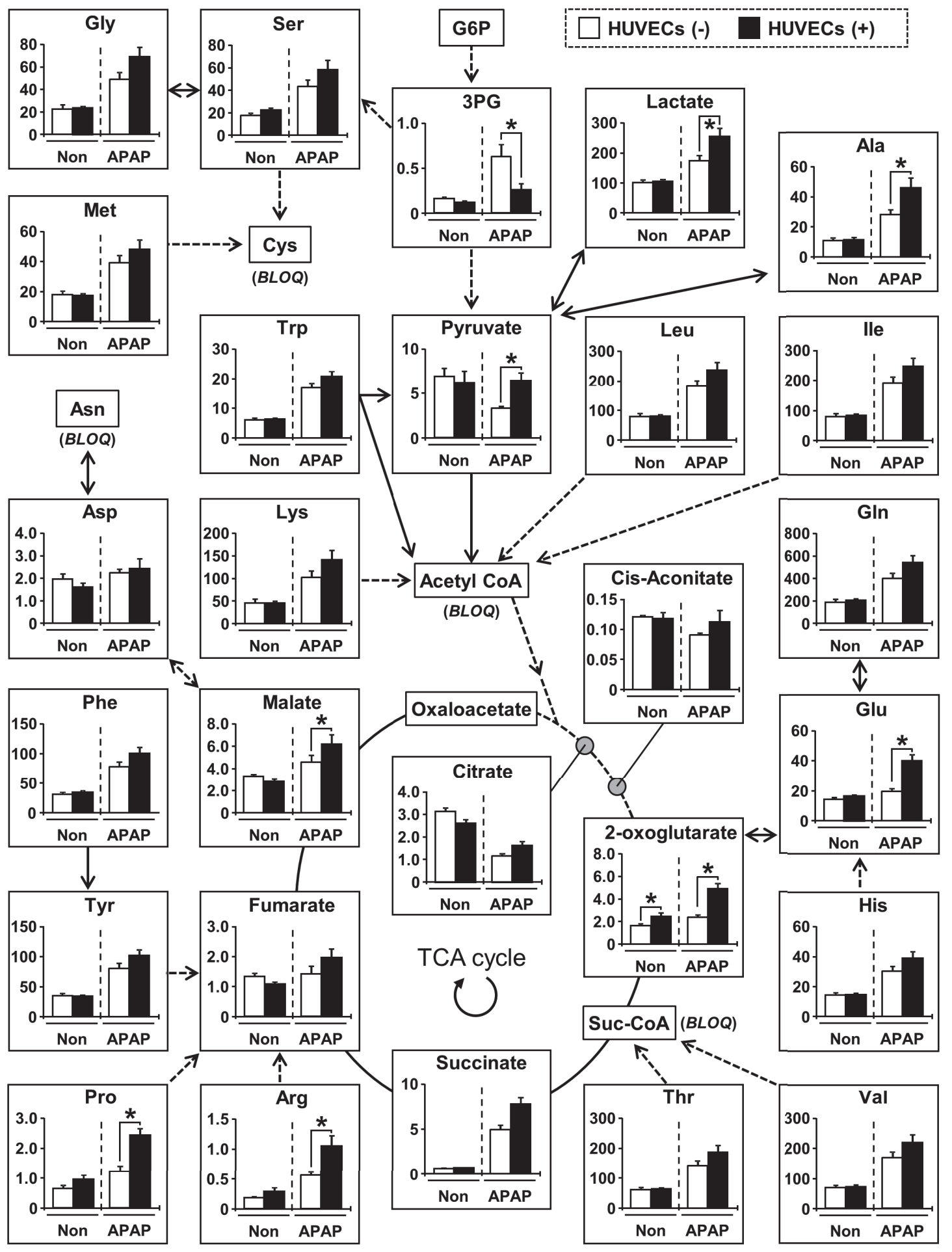

Fig. 3. Effect of acetaminophen on the levels of essential amino acids and major metabolites in the glycolysis and TCA pathways in the $\mathrm{IVL}_{\mathrm{EHS}}$ and monocultures. The y-axis shows relative metabolite levels (nmol/ $\mu \mathrm{g}$ of albumin). White and black columns indicate control and $\mathrm{IVL}_{\mathrm{EHS}}$, respectively. Non, no treatment; APAP, acetaminophen; $B L O Q$, metabolite level below the limitation of quantification. Data are expressed as the mean \pm S.E.M. $n=4$. Statistical analyses for significant differences were performed according to Student's $t$ test $(*, P<0.05$ compared to control). 
the TCA intermediates, an exception was 2-oxoglutarate (also known as $\alpha$-ketoglutarate) whose level was higher in the $\mathrm{IVL}_{\mathrm{EHS}}$ than in control cultures, and difference was even more pronounced after acetaminophen treatment (Fig. 3). Considering the role of 2-oxoglutarate as an antioxidant involved in reactive oxygen species (ROS) scavenging (Mailloux et al., 2007), the increase in 2-oxoglutarate levels could be attributed to increased tolerance of hepatocytes in the IVL $\mathrm{EHS}_{\mathrm{EHS}}$ to oxidative stress (Fig. 2b).

Recent studies show that the activation of autophagy protects against acetaminophen-induced hepatotoxicity in vivo (Lin et al., 2014; Igusa et al., 2012; Ni et al., 2012); therefore the pool of amino acids should be increased in the presence of acetaminophen as a result of autophagydependent proteolysis, which should contribute to cellular energy balance. Consistently, the increase in amino acid levels after acetaminophen treatment tended to be higher in the IVL $\mathrm{EHS}_{\mathrm{EHS}}$ than in control cultures (Fig. 3), suggesting that the $\mathrm{IVL}_{\mathrm{EHS}}$ may be superior to hepatocyte monocultures in reproducing the physiological conditions characteristic for the liver.

\section{Bile acids}

Bile acid secretion is one of the important functions of the liver. Therefore, we examined the effect of $\mathrm{IVL}_{\mathrm{EHS}}$ construction on the synthesis of bile acids by ana-

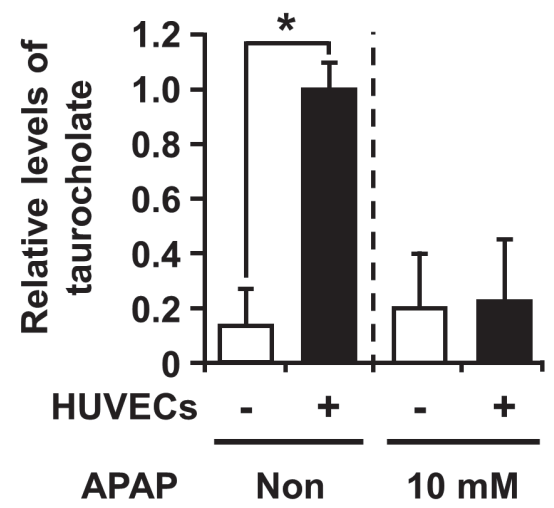

Fig. 4. Taurocholate levels in the $\mathrm{IVL}_{\mathrm{EHS}}$ and monocultures. Hepatocytes cultures were treated or not with $10 \mathrm{mM}$ acetaminophen (APAP) for $12 \mathrm{hr}$ and analyzed for intracellular taurocholate levels. Data are expressed as the mean \pm S.E.M. $n=4$. Except for the $\mathrm{IVL}_{\mathrm{EHS}}$ (with HUVECs) in the absence of acetaminophen treatment, each group contained the data which was below the limitation of quantification and thereby treated as zero. Statistical analyses for significant differences were performed according to Student's $t$ test $(*, P<0.05$ compared to control). lyzing the primary bile acid cholate and two conjugated bile acids, glycocholate and taurocholate. Although the levels of cholate and glycocholate in cell extracts were below the detection limit, taurocholate was identified and its production in the absence of acetaminophen treatment was higher in the $\mathrm{IVL}_{\mathrm{EHS}}$ than in control cultures (Fig. 4). This finding shows a possibility that $\mathrm{IVL}_{\mathrm{EHS}}$ system would contribute to maintain some metabolic functions in hepatocytes that decline in vitro even on the EHS gel. Our previous study revealed that bile canalicular membrane transporters involved in biliary excretion of hepatic metabolites showed higher or similar expression in the $\mathrm{IVL}_{\mathrm{EHS}}$ compared to hepatocyte monocultures on the EHS gel (Toyoda et al., 2012). Hence, the higher levels of intracellular taurocholate in the $\mathrm{IVL}_{\mathrm{EHS}}$ should be due to relatively increased/maintained taurocholate synthesis rather than decreased excretion. This interpretation is supported by the higher activities of major cytochrome $\mathrm{P} 450$ isozymes in $\mathrm{IVL}_{\mathrm{EHS}}$ as compared with those in monoculture control (Toyoda et al., 2012), since such enzyme-mediated oxidation is involved in the bile acid synthesis in the liver.

\section{Further consideration}

In the present study, we compared the metabolic activity of hepatocytes in the IVL $\mathrm{EHS}_{\mathrm{EHS}}$ and in monocultures on the EHS gel by analyzing the urea cycle, glycolysis, the TCA pathway, and production of essential amino acids. To the best of our knowledge, this is the first metabolomics study on the $\mathrm{IVL}_{\mathrm{EHS}}$. Since our study was limited in the metabolomics of cellular extracts that were prepared $36 \mathrm{hr}$ after the hepatocytes seeding, the long-term effects of the IVL $\mathrm{IHH}_{\mathrm{EHS}}$ construct on the metabolic profile of hepatocytes should be addressed in future investigation. However, our results could support the utility of $\mathrm{IVL}_{\mathrm{EHS}}$ in the short-term culture experiments. Although all metabolites were not quantified completely because of the detection limit, our results showed that the metabolic profile of hepatocytes was not affected by the $\mathrm{IVL}_{\mathrm{EHS}}$ construction; moreover, the $\mathrm{IVL}_{\mathrm{EHS}}$ rather supported hepatocyte metabolism in the presence of exogenous stress. These findings are in agreement with our previous studies on the mouse embryonic stem cell-derived liver tissue model consisting of a hepatocyte layer and surrounding sinusoid-like vascular endothelial cells (Tamai et al., 2013; Tsutsui et al., 2006; Ogawa et al., 2005), and support the pivotal role of the intercellular communication between hepatocytes and endothelial cells in the functional activity of hepatocytes.

Prior work has documented the effectiveness of $\mathrm{IVL}_{\mathrm{EHS}}$ containing murine hepatocytes and HUVEC network in the maintenance of hepatic functions (Toyoda et 
Metabolomics of an in vitro liver model

al., 2012). In this study we carried out the metabolomics of $\mathrm{IVL}_{\mathrm{EHS}}$ that was constructed by the same experimental procedure to extend our understanding about the biochemical features of this new culture system. On the other hand, this approach did not allow us to avoid an experimental mismatch in host species of the utilized cells in the $\mathrm{IVL}_{\mathrm{EHS}}$. Future study should therefore include the analysis of monospecific $\mathrm{IVL}_{\mathrm{EHS}}$.

In conclusion, our data strongly suggest that the $\mathrm{IVL}_{\mathrm{EHS}}$ construct could contribute to the maintenance of hepatocyte functions without negative effects on their metabolic status, and would be a better model than the exiting in vitro monoculture systems, both for investigating physiological mechanisms in the liver and for compound hepatotoxicity screening. Further transcriptomic and proteomic studies are required to confirm the utility of the IVL $\mathrm{L}_{\mathrm{EHS}}$ system as a reliable in vitro model of the liver.

\section{ACKNOWLEDGMENTS}

This study was supported by Japan Society for the Promotion of Science (JSPS) (Grants-in-Aid for Scientific Research [KAKENHI] number 09J09415 and 15H05610 to Y.T., and 21300178 and 25242040 to Y-I.T.), the Ministry of Education, Culture, Sports, Science and Technology (MEXT) (KAKENHI Grant number 23119003 to Y-I.T.) and Japan Agency for Medical Research and Development (AMED to Y-I.T. and AMED-CREST to T.S.). Y.T. was a JSPS research fellow.

Conflict of interest---- The authors declare that there is no conflict of interest.

\section{REFERENCES}

Ahn, S., Tamai, M., Nakashima, K., Ito, M., Suzuki, T. and Tagawa, Y. (2014): An in vitro liver model consisting of endothelial vascular networks surrounded by human hepatoma cell lines allows for improved hepatitis B virus replication. J. Biosci. Bioeng., 118, 107-111.

Atkinson, D.E. (1968): The energy charge of the adenylate pool as a regulatory parameter. Interaction with feedback modifiers. Biochemistry, 7, 4030-4034.

Bhatia, S.N., Balis, U.J., Yarmush, M.L. and Toner, M. (1998): Microfabrication of hepatocyte/fibroblast co-cultures: role of homotypic cell interactions. Biotechnol. Prog., 14, 378-387.

Bhatia, S.N., Balis, U.J., Yarmush, M.L. and Toner, M. (1999): Effect of cell-cell interactions in preservation of cellular phenotype: cocultivation of hepatocytes and nonparenchymal cells. FASEB J., 13, 1883-1900.

Chapman, A.G., Fall, L. and Atkinson, D.E. (1971): Adenylate energy charge in Escherichia coli during growth and starvation. J. Bacteriol., 108, 1072-1086.

Coen, M. (2015): Metabolic phenotyping applied to pre-clinical and clinical studies of acetaminophen metabolism and hepatotoxicity. Drug Metab. Rev., 47, 29-44.

Dalton, T.P., Shertzer, H.G. and Puga, A. (1999): Regulation of gene expression by reactive oxygen. Annu. Rev. Pharmacol. Toxicol., 39, 67-101.

de Nadal, E., Ammerer, G. and Posas, F. (2011): Controlling gene expression in response to stress. Nat. Rev. Genet., 12, 833-845.

Debray, F.G., Mitchell, G.A., Allard, P., Robinson, B.H., Hanley, J.A. and Lambert, M. (2007): Diagnostic accuracy of blood lactate-to-pyruvate molar ratio in the differential diagnosis of congenital lactic acidosis. Clin. Chem., 53, 916-921.

DiPersio, C.M., Jackson, D.A. and Zaret, K.S. (1991): The extracellular matrix coordinately modulates liver transcription factors and hepatocyte morphology. Mol. Cell Biol., 11, 4405-4414.

Donato, M.T., Jover, R. and Gómez-Lechón, M.J. (2013): Hepatic cell lines for drug hepatotoxicity testing: limitations and strategies to upgrade their metabolic competence by gene engineering. Curr. Drug Metab., 14, 946-968.

Gissen, P. and Arias, I.M. (2015): Structural and functional hepatocyte polarity and liver disease. J. Hepatol., 63, 1023-1037.

Godoy, P., Hewitt, N.J., Albrecht, U., Andersen, M.E., Ansari, N., Bhattacharya, S., Bode, J.G., Bolleyn, J., Borner, C., Böttger, J., Braeuning, A., Budinsky, R.A., Burkhardt, B., Cameron, N.R., Camussi, G., Cho, C.S., Choi, Y.J., Craig Rowlands, J., Dahmen, U., Damm, G., Dirsch, O., Donato, M.T., Dong, J., Dooley, S., Drasdo, D., Eakins, R., Ferreira, K.S., Fonsato, V., Fraczek, J., Gebhardt, R., Gibson, A., Glanemann, M., Goldring, C.E., Gómez-Lechón, M.J., Groothuis, G.M., Gustavsson, L., Guyot, C., Hallifax, D., Hammad, S., Hayward, A., Häussinger, D., Hellerbrand, C., Hewitt, P., Hoehme, S., Holzhütter, H.G., Houston, J.B., Hrach, J., Ito, K., Jaeschke, H., Keitel, V., Kelm, J.M., Kevin Park, B., Kordes, C., Kullak-Ublick, G.A., LeCluyse, E.L., Lu, P., Luebke-Wheeler, J., Lutz, A., Maltman, D.J., Matz-Soja, M., McMullen, P., Merfort, I., Messner, S., Meyer, C., Mwinyi, J., Naisbitt, D.J., Nussler, A.K., Olinga, P., Pampaloni, F., Pi, J., Pluta, L., Przyborski, S.A., Ramachandran, A., Rogiers, V., Rowe, C., Schelcher, C., Schmich, K., Schwarz, M., Singh, B., Stelzer, E.H., Stieger, B., Stöber, R., Sugiyama, Y., Tetta, C., Thasler, W.E., Vanhaecke, T., Vinken, M., Weiss, T.S., Widera, A., Woods, C.G., Xu, J.J., Yarborough, K.M. and Hengstler, J.G. (2013): Recent advances in 2D and 3D in vitro systems using primary hepatocytes, alternative hepatocyte sources and non-parenchymal liver cells and their use in investigating mechanisms of hepatotoxicity, cell signaling and ADME. Arch. Toxicol., 87, 1315-1530.

Guguen-Guillouzo, C. and Guillouzo, A. (2010): General review on in vitro hepatocyte models and their applications. Methods Mol. Biol., 640, 1-40.

Hewitt, N.J., Lechón, M.J., Houston, J.B., Hallifax, D., Brown, H.S., Maurel, P., Kenna, J.G., Gustavsson, L., Lohmann, C., Skonberg, C., Guillouzo, A., Tuschl, G., Li, A.P., LeCluyse, E., Groothuis, G.M. and Hengstler, J.G. (2007): Primary hepatocytes: current understanding of the regulation of metabolic enzymes and transporter proteins, and pharmaceutical practice for the use of hepatocytes in metabolism, enzyme induction, transporter, clearance, and hepatotoxicity studies. Drug Metab. Rev., 39, 159-234.

Hirayama, A., Kami, K., Sugimoto, M., Sugawara, M., Toki, N., Onozuka, H., Kinoshita, T., Saito, N., Ochiai, A., Tomita, M., Esumi, H. and Soga, T. (2009): Quantitative metabolome profiling of colon and stomach cancer microenvironment by capillary electrophoresis time-of-flight mass spectrometry. Cancer Res., 
69, 4918-4925.

Igusa, Y., Yamashina, S., Izumi, K., Inami, Y., Fukada, H., Komatsu, M., Tanaka, K., Ikejima, K. and Watanabe, S. (2012): Loss of autophagy promotes murine acetaminophen hepatotoxicity. J. Gastroenterol., 47, 433-443.

Jaeschke, H., Knight, T.R. and Bajt, M.L. (2003): The role of oxidant stress and reactive nitrogen species in acetaminophen hepatotoxicity. Toxicol. Lett., 144, 279-288.

Kaplowitz, N. (2005): Idiosyncratic drug hepatotoxicity. Nat. Rev. Drug Discov., 4, 489-499.

Kidambi, S., Yarmush, R.S., Novik, E., Chao, P., Yarmush, M.L. and Nahmias, Y. (2009): Oxygen-mediated enhancement of primary hepatocyte metabolism, functional polarization, gene expression, and drug clearance. Proc. Natl. Acad. Sci. USA, 106, 1571415719.

Kleinman, H.K. and Martin, G.R. (2005): Matrigel: basement membrane matrix with biological activity. Semin. Cancer Biol., 15, 378-386.

Kleinman, H.K., Philp, D. and Hoffman, M.P. (2003): Role of the extracellular matrix in morphogenesis. Curr. Opin. Biotechnol., 14, 526-532.

Lancaster, E.M., Hiatt, J.R. and Zarrinpar, A. (2015): Acetaminophen hepatotoxicity: an updated review. Arch. Toxicol., 89, 193-199.

Lin, S.J. and Guarente, L. (2003): Nicotinamide adenine dinucleotide, a metabolic regulator of transcription, longevity and disease. Curr. Opin. Cell Biol., 15, 241-246.

Lin, Z., Wu, F., Lin, S., Pan, X., Jin, L., Lu, T., Shi, L., Wang, Y., $\mathrm{Xu}, \mathrm{A}$. and Li, X. (2014): Adiponectin protects against acetaminophen-induced mitochondrial dysfunction and acute liver injury by promoting autophagy in mice. J. Hepatol., 61, 825-831.

Mailloux, R.J., Bériault, R., Lemire, J., Singh, R., Chénier, D.R., Hamel, R.D. and Appanna, V.D. (2007): The tricarboxylic acid cycle, an ancient metabolic network with a novel twist. PLoS One, 2, e690.

Nahmias, Y., Schwartz, R.E., Hu, W.S., Verfaillie, C.M. and Odde, D.J. (2006): Endothelium-mediated hepatocyte recruitment in the establishment of liver-like tissue in vitro. Tissue Eng., 12, 1627-1638.

Ni, H.M., Bockus, A., Boggess, N., Jaeschke, H. and Ding, W.X. (2012): Activation of autophagy protects against acetaminopheninduced hepatotoxicity. Hepatology, 55, 222-232.

Ogawa, S., Tagawa, Y., Kamiyoshi, A., Suzuki, A., Nakayama, J., Hashikura, Y. and Miyagawa, S. (2005): Crucial roles of mesodermal cell lineages in a murine embryonic stem cell-derived in vitro liver organogenesis system. Stem Cells, 23, 903-913.

Schuetz, E.G., Li, D., Omiecinski, C.J., Muller-Eberhard, U., Kleinman, H.K., Elswick, B. and Guzelian, P.S. (1988): Regulation of gene expression in adult rat hepatocytes cultured on a basement membrane matrix. J. Cell Physiol., 134, 309-323.

Sharma, R., Greenhough, S., Medine, C.N. and Hay, D.C. (2010): Three-dimensional culture of human embryonic stem cell derived hepatic endoderm and its role in bioartificial liver construction. J. Biomed. Biotechnol., 2010, 236147.

Soga, T., Baran, R., Suematsu, M., Ueno, Y., Ikeda, S., Sakurakawa, T., Kakazu, Y., Ishikawa, T., Robert, M., Nishioka, T. and Tomita, M. (2006): Differential metabolomics reveals ophthalmic acid as an oxidative stress biomarker indicating hepatic glutathione consumption. J. Biol. Chem., 281, 16768-16776.

Soga, T., Igarashi, K., Ito, C., Mizobuchi, K., Zimmermann, H.P. and Tomita, M. (2009): Metabolomic profiling of anionic metabolites by capillary electrophoresis mass spectrometry. Anal. Chem., 81, 6165-6174.

Soga, T., Ohashi, Y., Ueno, Y., Naraoka, H., Tomita, M. and Nishioka, T. (2003): Quantitative metabolome analysis using capillary electrophoresis mass spectrometry. J. Proteome Res., 2, 488-494.

Soga, T., Ueno, Y., Naraoka, H., Ohashi, Y., Tomita, M. and Nishioka, T. (2002): Simultaneous determination of anionic intermediates for Bacillus subtilis metabolic pathways by capillary electrophoresis electrospray ionization mass spectrometry. Anal. Chem., 74, 2233-2239.

Strain, A.J. (1999): Ex vivo liver cell morphogenesis: one step nearer to the bioartificial liver? Hepatology, 29, 288-290.

Sun, F., Dai, C., Xie, J. and Hu, X. (2012): Biochemical issues in estimation of cytosolic free NAD/NADH ratio. PLoS One, 7, e34525.

Tamai, M., Aoki, M., Nishimura, A., Morishita, K. and Tagawa, Y. (2013): In vitro recapitulation of the urea cycle using murine embryonic stem cell-derived in vitro liver model. Amino Acids, 45, 1343-1351.

Toyoda, Y., Tamai, M., Kashikura, K., Kobayashi, S., Fujiyama, Y., Soga, T. and Tagawa, Y. (2012): Acetaminophen-Induced Hepatotoxicity in a Liver Tissue Model Consisting of Primary Hepatocytes Assembling around an Endothelial Cell Network. Drug Metab. Dispos., 40, 169-177.

Tsutsui, M., Ogawa, S., Inada, Y., Tomioka, E., Kamiyoshi, A., Tanaka, S., Kishida, T., Nishiyama, M., Murakami, M., Kuroda, J., Hashikura, Y., Miyagawa, S., Satoh, F., Shibata, N. and Tagawa, Y. (2006): Characterization of cytochrome P450 expression in murine embryonic stem cell-derived hepatic tissue system. Drug Metab. Dispos., 34, 696-701.

Viollet, B., Guigas, B., Leclerc, J., Hébrard, S., Lantier, L., Mounier, R., Andreelli, F. and Foretz, M. (2009): AMP-activated protein kinase in the regulation of hepatic energy metabolism: from physiology to therapeutic perspectives. Acta. Physiol. (Oxf), 196, 81-98.

Williamson, D.H., Lund, P. and Krebs, H.A. (1967): The redox state of free nicotinamide-adenine dinucleotide in the cytoplasm and mitochondria of rat liver. Biochem. J., 103, 514-527.

Zhang, L. and Vertes, A. (2015): Energy Charge, Redox State, and Metabolite Turnover in Single Human Hepatocytes Revealed by Capillary Microsampling Mass Spectrometry. Anal. Chem., 87, 10397-10405. 